\title{
ON CERTAIN TOPOLOGICAL INDICES OF BENZENOID COMPOUNDS
}

\author{
S. Prabhu ${ }^{1^{*}}$, M. Arulperumjothi ${ }^{2}$ \\ ${ }^{1}$ Department of Applied Mathematics Sri Venkateswara College of Engineering, Sriperumbudur, India 602105 \\ drprabhu@svce.ac.in \\ ${ }^{2}$ Department of Mathematics, Loyola College, Chennai, India 600034 \\ marulperumjothi@gmail.com \\ ${ }^{*}$ corresponding author
}

\section{ABSTRACT}

Drug discovery is mainly the result of chance discovery and massive screening of large corporate libraries of synthesized or naturally-occurring compounds. Computer aided drug design is an approach to rational drug design made possible by the recent advances in computational chemistry in various fields of chemistry, such as molecular graphics, molecular mechanics, quantum chemistry, molecular dynamics, library searching, prediction of physical, chemical, and biological properties. The structure of a chemical compound can be represented by a graph whose vertex and edge specify the atom and bonds respectively. Topological indices are designed basically by transforming a molecular graph into a number. A topological index is a numeric quantity of a molecule that is mathematically derived from the structural graph of a molecule. In this paper we compute certain topological indices of pyrene molecular graph. The topological indices are used in quantitative structure-property relationships (QSPR) and quantitative structure-activity relationships (QSAR) studies.

\section{Keywords}

Topological indices, molecular graph, pyrene, wiener index, gutman index, zagreb index, structure-property, structureactivity

\section{Academic Discipline And Sub-Disciplines}

Mathematical Chemistry, Computational Chemistry, Molecular Chemistry, Medicinal Chemistry.

\section{SUBJECT CLASSIFICATION}

Mathematics Subject Classification: 05C05, 05C07, 05C35

\section{INTRODUCTION}

Let $G(V, E)$ be a connected molecular graph. The distance between two distinct vertices $u, v \in V(G)$, denoted by $d(u, v)$ is the length of a shortest $u-v$ path in $G$. Topological indices are designed basically by transforming a molecular graph into a number. The concept of topological index came from work done by Harold Wiener in 1947 while he was working on boiling point of paraffin [1]. The Wiener index of $G$ is defined as $W(G)=\sum_{\left[u_{2} v\right] \subseteq V(G)} d(u, v)$. Two important topological indices introduced about forty years ago by Ivan Gutman and Trinajstic [2] are the first zagreb index $M_{1}(G)$ and the second zagreb index $M_{2}(G)$ which are defined as

$$
\begin{aligned}
& M_{1}(G)=\sum_{w v \in E(G)}\left[d_{u}+d_{v}\right] \\
& M_{2}(G)=\sum_{u v \in E(G)}\left[d_{u} \times d_{v}\right]
\end{aligned}
$$

where $d_{u}$ denotes the degree of $u$.

The degree distance was introduced by Dobrynin and Kochetova [3] and the weighted version of the Wiener index [4] is introduced by Gutman in 1994. The degree distance of $G$ and Gutman index of $G$ respectively defined and denoted by

$$
\begin{array}{r}
D D(G)=\sum_{\{u, v] \subseteq V(G)} d(u, v) \times\left[d_{u}+d_{v}\right] \\
\operatorname{Gut}(G)=\sum_{\{u, v] \subseteq V(G)} d(u, v) \times d_{u} \times d_{v}
\end{array}
$$




\section{MATERIAL AND METHODS}

Pyrene is a polycyclic aromatic hydrocarbon consisting of four fused benzene rings, resulting in a flat aromatic system. The chemical formula is $\mathrm{C}_{16} \mathrm{H}_{10}$. This colorless solid is the smallest peri-fused $\mathrm{PAH}$. It is one of a group of chemicals called polycyclic aromatic hydrocarbons, PAHs for short. PAHs are often found together in groups of two or more. They can exist in over 100 different combinations but the most common are treated as a group of 15 . PAHs are found naturally in the environment but they can also be man-made. Pyrene is colorless crystal-like solid but can also look yellow. PAHs are created when products like coal, oil, gas, and garbage is burned but the burning process is not complete.

Not much information is available on the individual chemicals within the PAH group. Most of the information available is for the PAH group as a whole. Information specific to pyrene is included in this fact sheet when available. Most of the PAHs are used to conduct research. Like most PAHs, pyrene is used to make dyes, plastics and pesticides. It has also been used to make other PAHs called benzo(a)pyrene, benzo(e)pyrene and $2 \mathrm{H}$-benzo(cf)pyrene-2. See Figure 1.

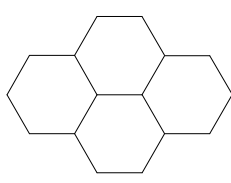

(i)

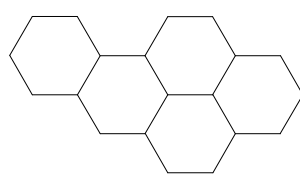

(ii)

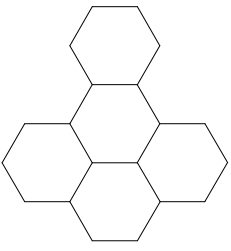

(iii)

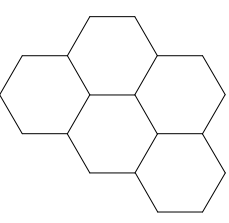

(iv)

Fig 1: (i) pyrene ; (ii) benzo (a) pyrene ; (iii) benzo (e) pyrene ; (iv) 2H-benzo (cf) pyrene-2

Benzo[a]pyrene is a polycyclic aromatic hydrocarbon found in coal tar with the formula $\mathrm{C}_{20} \mathrm{H}_{12}$. Its metabolites are mutagenic and highly carcinogenic, and it is listed as a Group 1 carcinogen by the International Agency for Research on Cancer (IARC). Benzo[e]pyrene is a polycyclic aromatic hydrocarbon with the chemical formula $\mathrm{C}_{20} \mathrm{H}_{12}$. It is listed as a Group 3 carcinogen by the IARC. Resistomycin $(6 \mathrm{Cl})$, Antibiotic A 3733A, Geliomycin, Itamycin are the other names of $2 \mathrm{H}-$ Benzo[cd]pyrene-2.

\section{RESULTS}

Pyrene $P Y(n)$ has $2 n^{2}+4 n$ vertices and $3 n^{2}+4 n-1$ edges and it is bipartite as this does not contain odd cycle. See Figure. 2. The bondage number, packing of pyrene and pyrene torus is discussed in [5].
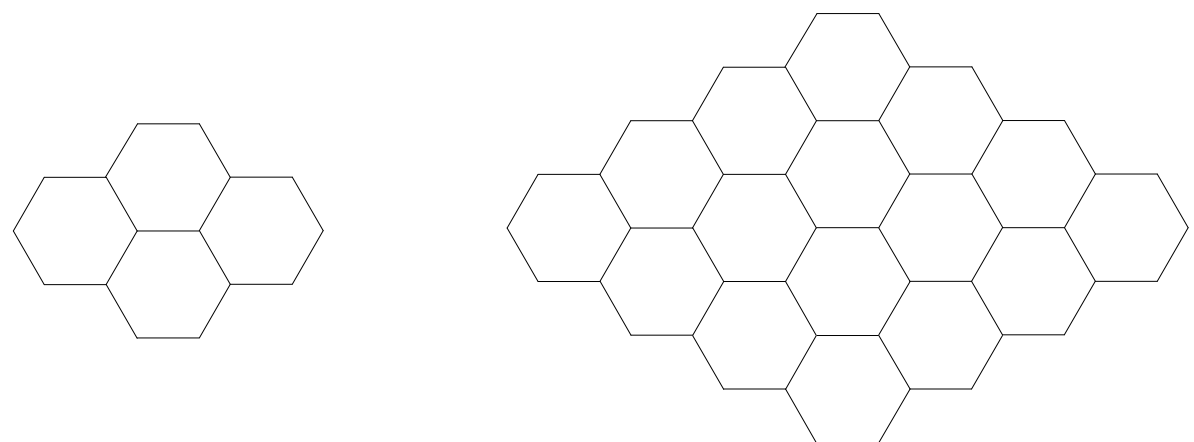

Fig 2: Molecular graph of PY(2) and PY(4)

\section{Zagreb Indices}

The first and second Zagreb indices were first introduced by I. Gutman and N. Trinajstic in 1972. It is reported that these indices are useful in the study of anti-inflammatory activities of certain chemical instances, and in elsewhere. Recently, the first and second multiple Zagreb indices of a graph $G$ were introduced by Ghorbani and Azimi in 2012. The Zagreb indices were first appeared in the topological formula for the total $\pi$-energy of conjugated molecules that has been derived in 1972 by Gutman and Trinajstic [2]. These indices have also been used as branching indices [6] and have found applications in QSPR and QSAR studies.

The first Zagreb index $M_{1}(G)$ and the second Zagreb index $M_{2}(G)$ of a molecular graph $G$ are respectively defined as

$$
M_{1}(G)=\sum_{u v \in E(G)}\left[d_{u}+d_{v}\right]
$$




$$
M_{2}(G)=\sum_{u v \in E(G)}\left[d_{u} \times d_{v}\right]
$$

The new multiplicative versions of $M_{1}(G)$ and $M_{2}(G)$ indices, denoted by $P M_{1}(G)$ and $P M_{2}(G)$ (respectively), were first defined by Ghorbani and Azimi [7]. These indices are obtained for $\mathrm{TiO}_{2}$ Nanotubes in [8] and defined as follows.

$$
\begin{aligned}
& P M_{1}(G)=\prod_{u v \in E(G)}\left[d_{u}+d_{v}\right] \\
& P M_{1}(G)=\prod_{u v \in E(G)}\left[d_{u} \times d_{v}\right]
\end{aligned}
$$

Theorem 3.1. Let $G$ be a molecular graph of $P Y(n), n \geq 2$. Then

$$
\begin{gathered}
M 1(G)=18 n^{2}+16 n-10 \\
M 2(G)=27 n^{2}+12 n-15 \\
P M_{1}(G)=\frac{24576}{390625} \times 301.4082^{n} \times 216^{n^{2}} \\
P M_{2}(G)=\frac{36864}{1679616} \times 256^{n} \times 729^{n^{2}}
\end{gathered}
$$

Proof. Let $V_{1}(G)$ and $V_{2}(G)$ be the vertex partition of $G$ and $V_{1}(G)$ and $V_{2}(G)$ be the set of all 2-degree and 3-degree vertices respectively. $\left|V_{1}(G)\right|=4 n+2$ and $\left|V_{2}(G)\right|=2 n^{2}-2$. Let $E_{1}(G), E_{2}(G)$ and $E_{3}(G)$ be the three partition of edges of $E(G)$. The edges in $E_{1}(G)$ is incident with both 2-degree vertices, the edges in $E_{2}(G)$ is incident with one 2-degree vertex and one 3-degree vertex and the edges in $E_{3}(G)$ is incident with both 3-degree vertices. $\left|E_{1}(G)\right|=6,\left|E_{2}(G)\right|=8 n-8$ and $\left|E_{3}(G)\right|=3 n^{2}-4 n+1$.

$$
\begin{aligned}
M_{1}(G)=\sum_{u v \in E(G)}\left[d_{u}+d_{v}\right] & \\
= & \sum_{u v \in E_{1}(G)}\left[d_{u}+d_{v}\right]+\sum_{u v E E_{2}(G)}\left[d_{u}+d_{v}\right] \\
& +\sum_{u v \in E_{a}(G)}\left[d_{u}+d_{v}\right]=6 \times[2+2]+(8 n-2) \times[2+3]+\left(3 n^{2}-4 n+1\right) \\
& \times[3+3]=18 n^{2}+16 n-10 . \\
M_{2}(G)=\sum_{u v \in E(G)}\left[d_{u} \times d_{v}\right] & =\sum_{u v \in E_{1}(G)}\left[d_{u} \times d_{v}\right]+\sum_{u v \in E_{2}(G)}\left[d_{u} \times d_{v}\right] \\
& +\sum_{u v \in E_{s}(G)}\left[d_{u} \times d_{v}\right]=6 \times[2 \times 2]+(8 n-2) \times[2 \times 3]+\left(3 n^{2}-4 n+1\right) \\
& \times[3 \times 3]=27 n^{2}+12 n-15 .
\end{aligned}
$$




$$
\begin{aligned}
& P M_{1}(G)=\prod_{u v \in E(G)}\left[d_{u}+d_{v}\right] \\
& =\prod_{u v \in E_{1}(G)}\left[d_{u}+d_{v}\right] \times \prod_{u v \in E_{2}(G)}\left[d_{u}+d_{v}\right] \times \prod_{u v \in E_{g}(G)}\left[d_{u}+d_{v}\right] \\
& =\underbrace{[2+2] \times[2+2] \ldots \times[2+2]}_{6 \text { times }} \times \underbrace{[2+3] \times[2+3] \ldots \times[2+3]}_{8 n-8 \text { timess }} \\
& \times \underbrace{[3+3] \times[3+3] \times \ldots[3+3]}_{\left(3 n^{2}-4 n+1\right) \text { times }}=\frac{24576}{390625} \times 301.4082^{n} \times 216^{n^{2}} \\
& P M_{2}(G)=\prod_{u v \in E(G)}\left[d_{u} \times d_{v}\right] \\
& =\prod_{u v \in E_{1}(G)}\left[d_{u} \times d_{v}\right] \times \prod_{u v \in E_{2}(G)}\left[d_{u} \times d_{v}\right] \times \prod_{u v E E_{g}(G)}\left[d_{u} \times d_{v}\right] \\
& =\underbrace{[2 \times 2] \times[2 \times 2] \ldots \times[2 \times 2]}_{6 \text { times }} \times \underbrace{[2 \times 3] \times[2+3] \ldots \times[2+3]}_{8 n-8 \text { timess }} \\
& \times \underbrace{[3 \times 3] \times[3 \times 3] \times \ldots[3 \times 3]}_{\left(3 n^{2}-4 n+1\right) \text { times }}=\frac{36864}{1679616} \times 256^{n} \times 729^{n^{2}}
\end{aligned}
$$

Figure 3 Depicts the comparative chart of first and second Zagreb indices with respect to the dimension of the pyrene.

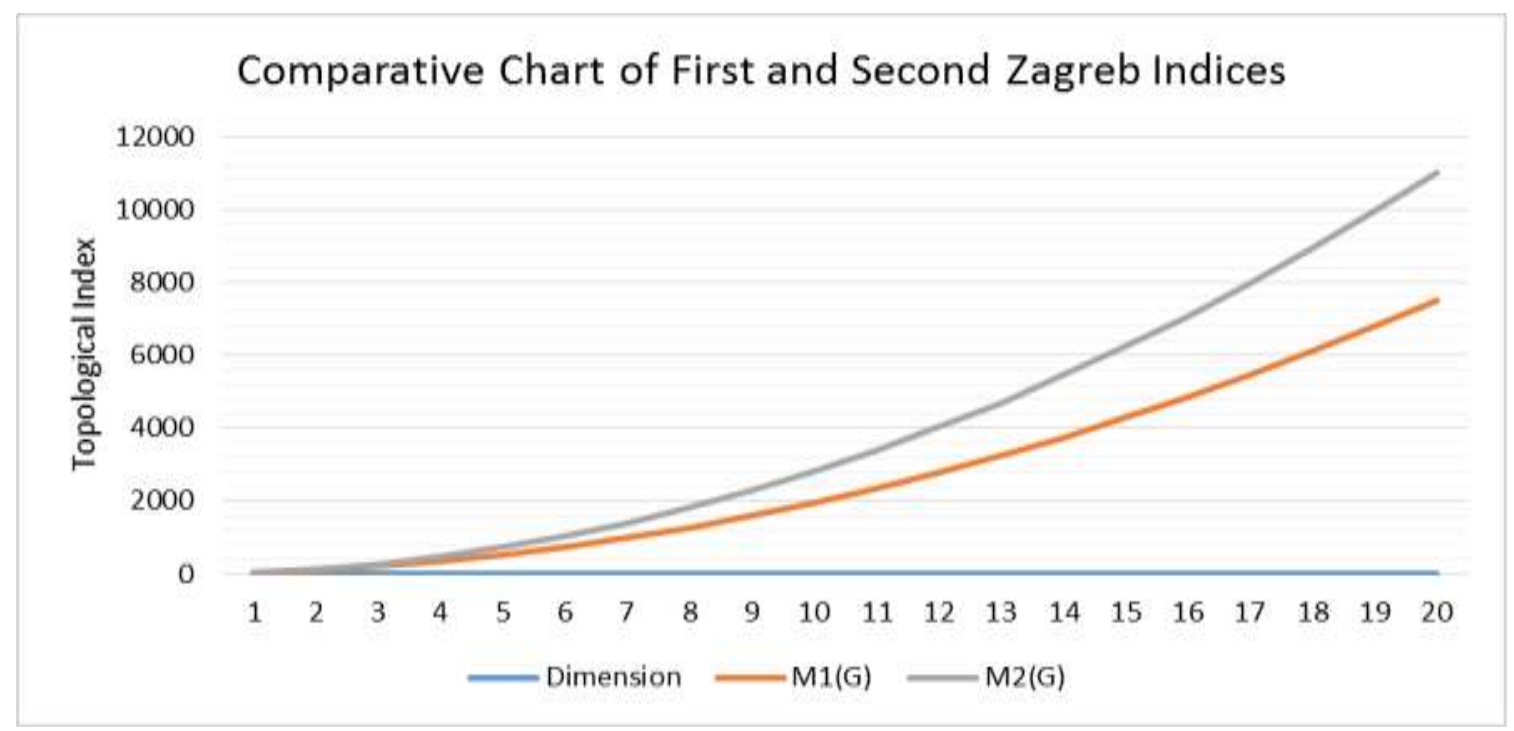

Figure 3: Comparative chart of first and second Zagreb indices.

\section{$A B C$ Index, GA index and Randi'c index}

The first geometric-arithmetic (GA) index was proposed by Vuki"cevi'c and Furtula [9]. It has been demonstrated, on the example of octane isomers, that GA index is well-correlated with a variety of physico-chemical properties [9]. The details about mathematical properties of the GA indices and their applications in QSPR and QSAR can be found in the survey [10].

$$
G A(G)=\sum_{u v \in E(G)} \frac{\sqrt{d_{u} \times d_{v}}}{\frac{1}{2} \times\left(d_{u}+d_{v}\right)}
$$

The atom-bond connectivity $A B C$ index of graph $G$ is proposed by Estrada et al. in [12] which is abbreviated as $A B C(G)$ and defined as 


$$
A B C(G)=\sum_{u v \in E(G)} \sqrt{\frac{d_{u}+d_{v}-2}{d_{u} \times d_{v}}}
$$

The $A B C$ index provides a good model for the stability of linear and branched alkanes as well as the strain energy of cycloalkanes [3,12]. Due to its physico-chemical applicability, the $A B C$ index has attracted significant attention from researchers in recent years and many mathematical properties of this index were reported in [12, 13, 14].

For a graph $G=(V, E)$, the general Randi'c index $R_{\alpha}(G)$ is defined as the sum of $\left(d_{u} \times d_{v}\right)^{\alpha}$ over all edges $e=u v$ of $G$.

$$
R_{\alpha}(G)=\sum_{w v \in E(G)}\left(d_{u} \times d_{v}\right)^{\alpha}
$$

The Randi'c index was introduced in [15] by Randi'c in 1975 with $\alpha=-0.5$ as one of the many graph-theoretical parameters derived from the graph underlying some molecule. This index has been closely correlated with many chemical properties. The general Randi'c index was proposed by Bollob'as and Erd"os [16], and Amic et al.[17], independently, in 1998. Then it has been extensively studied by both mathematicians and theoretical chemists [18]. Many important mathematical properties have been established [19].

Theorem 3.2. Let $G$ be a molecular graph of $P Y(n), n \geq 2$. Then

$$
\begin{gathered}
G A(G)=\frac{1}{5}\left[15 n^{2}-(20-16 \sqrt{6}) n+35-16 \sqrt{6}\right] \\
A B C(G)=\frac{1}{3}\left[6 n^{2}+(12 \sqrt{2}-8) n+6 \sqrt{2}+2\right] \\
R_{\frac{1}{2}}(G)=\frac{1}{3}\left(3 n^{2}-4 n+1\right)+\frac{1}{\sqrt{6}}(8 n-8)+3
\end{gathered}
$$

Proof. Let $E_{1}(G), E_{2}(G)$ and $E_{3}(G)$ be the three partition of edges of $E(G)$. The edges in $E_{1}(G)$ is incident with both 2 degree vertices, The edges in $E_{2}(G)$ is incident with one 2-degree vertex and one 3-degree vertex and The edges in $E_{3}(G)$ is incident with both 3-degree vertices.

$$
\begin{aligned}
& G A(G)=\sum_{w v \in E(G)} \frac{\sqrt{d_{u} \times d_{v}}}{\frac{1}{2} \times\left(d_{u}+d_{v}\right)} \\
& =\sum_{w v \in E_{1}(G)} \frac{\sqrt{d_{u} \times d_{v}}}{\frac{1}{2} \times\left(d_{u}+d_{v}\right)}+\sum_{w v \in E_{2}(G)} \frac{\sqrt{d_{u} \times d_{v}}}{\frac{1}{2} \times\left(d_{u}+d_{v}\right)}+\sum_{w v \in E_{g}(G)} \frac{\sqrt{d_{u} \times d_{v}}}{\frac{1}{2} \times\left(d_{u}+d_{v}\right)} \\
& =6 \times \sum_{w V \in E_{1}(G)} \frac{\sqrt{2 \times 2}}{\frac{1}{2} \times(2+2)}+(8 n-8) \times \sum_{w V \in E_{n}(G)} \frac{\sqrt{2 \times 3}}{\frac{1}{2} \times(2+3)}+\left(3 n^{2}-4 n+1\right) \\
& \times \sum_{w \nu \in E_{a}(G)} \frac{\sqrt{3 \times 3}}{\frac{1}{2} \times(3+3)}=\frac{1}{5}\left[15 n^{2}-(20-16 \sqrt{6}) n+35-16 \sqrt{6}\right] \text {. }
\end{aligned}
$$

$$
\begin{aligned}
A B C(G)= & \sum_{w v \in E(G)} \sqrt{\frac{d_{u}+d_{v}-2}{d_{u} \times d_{v}}} \\
& =\sum_{w v \in E_{1}(G)} \sqrt{\frac{d_{u}+d_{v}-2}{d_{u} \times d_{v}}}+\sum_{w v \in E_{n}(G)} \sqrt{\frac{d_{u}+d_{v}-2}{d_{u} \times d_{v}}}+\sum_{w v \in E_{s}(G)} \sqrt{\frac{d_{u}+d_{v}-2}{d_{u} \times d_{v}}} \\
& =6 \times \sum_{w v \in E_{1}(G)} \sqrt{\frac{2+2-2}{2 \times 2}}+(8 n-8) \times \sum_{w v \in E_{2}(G)} \sqrt{\frac{2+3-2}{2 \times 3}+\left(3 n^{2}-4 n+1\right)} \\
& \times \sum_{w v \in E_{s}(G)} \sqrt{\frac{3+3-2}{3 \times 3}}=\frac{1}{3}\left[6 n^{2}+(12 \sqrt{2}-8) n+6 \sqrt{2}+2\right] .
\end{aligned}
$$




$$
\begin{aligned}
R_{\frac{1}{2}}(G)=\sum_{w v \in E(G)}\left(d_{u} \times d_{v}\right)^{-0.5} \\
=\sum_{w v \in E_{1}(G)}\left(d_{u} \times d_{v}\right)^{-0.5}+\sum_{w v \in E_{2}(G)}\left(d_{u} \times d_{v}\right)^{-0.5}+\sum_{w v \in E_{s}(G)}\left(d_{u} \times d_{v}\right)^{-0.5} \\
=6 \times \sum_{w v \in E_{1}(G)}(2 \times 2)^{-0.5}+(8 n-8) \times \sum_{w v \in E_{2}(G)}(2 \times 3)^{-0.5}+\left(3 n^{2}-4 n+1\right) \\
\quad \times \sum_{w v \in E_{\Omega}(G)}(3 \times 3)^{-0.5}=\frac{1}{3}\left(3 n^{2}-4 n+1\right)+\frac{1}{\sqrt{6}}(8 n-8)+3 .
\end{aligned}
$$

The following Figure 4 Explains the comparative study of the above derived topological indices.

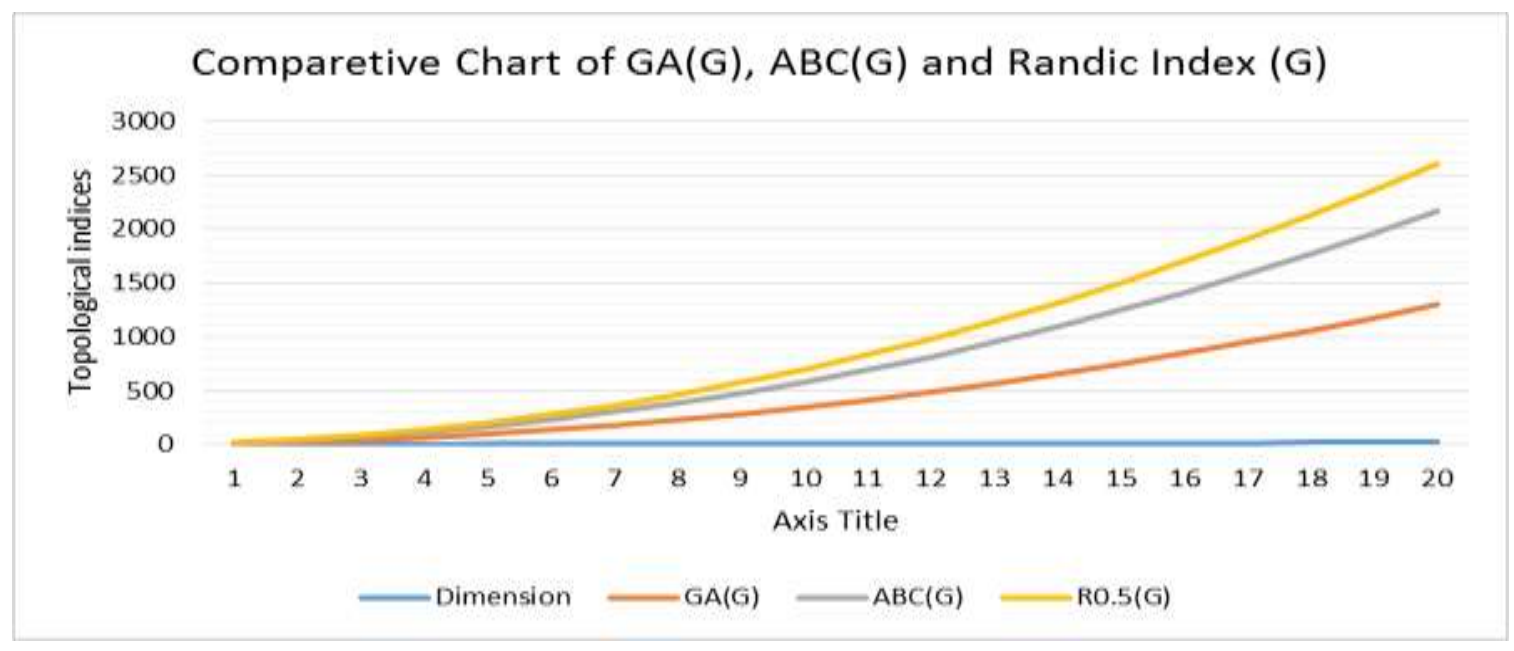

Figure 3: Comparative chart of $G A(G), A B C(G)$ and Randic Indices.

\section{CONCLUSION}

In this paper we have obtained some of the degree based topological indices of pyrene molecular graph. The other topological indices are under investigation.

\section{ACKNOWLEDGMENT}

The Second author is Supported by UGC-IGSGC Fellowship No. F. 8-1/2014-16/SGC-OBC- 2014-23907 / (SA-III), University Grants Commission, New Delhi, Government of India.

\section{REFERENCES}

1. H. Yousefi-Azari, M. H. Khalifeh, A. R. Ashra, Calculating the edge Wiener and Szeged indices of graphs, J. Comput. Appl. Math. 235 (2011) 4866-4870.

2. I. Gutman, selected Properties of the Schultz Molecular Topological Index, J. Chem. Inf. Comput. Sci., 34 (1994) 1087-1089.

3. Estrada, E., Torres, L., Rodrguez, L., and Gutman, I.: An atom-bond connectivity index: modelling the enthalpy of formation of alkanes, Indian J. Chem. A, 37 (1998) 849-855.

4. I. Gutman, S. Klavzar, An algorithm for the calculation of Szeged index of benzenoid hydrocarbons, J. Chem. Inf. Comput. Sci. 35 (1995) 1011-1014.

5. M. Randi'c, On characterization of molecular branching, J. Amer. Chem. Soc. 97 (1975) 6609-6615.

6. Dimitrov, D.: On structural properties of trees with minimal atom-bond connectivity index, Discr. Appl. Math.,172 (2014) 28-44.

7. I. Gutman, N. Trinajstic, Graph theory and molecular orbitals. Total $\pi$-electron energy of alternant hydrocarbons, Chem. Phys. Let., 17 (1972) 535-538.

8. M. Ali Malik and M. Imran, On the multiple zagreb indices of $\mathrm{TiO}_{2}$ nanotubes, Acta Chim. Slov.,62 (2015) 973976. 
9. H. Wiener, Structural determination of paraffin boiling points, J. Am. Chem. Soc., 69 (1947) 17-20.

10. M. V. Diudea, (Ed.), QSPR/QSAR Studies by Molecular Descriptors, NOVA, New York, (2001).

11. M. Ghorbani, N. Azimi, Iranian Journal of Mathematical Chemistry, 3 (2012) 137-143.

12. A. A. Dobrynin and A. A. Kochetova, Degree Distance of a Graph. A Degree Analogue of the Wiener Index, J. Chem. Inf. Comput. Sci., 34 (1994) 1082-1086.

13. Y. Hu, X. Li,Y. Shi, T. Xu, I. Gutman, On molecular graphs with smallest and greatest zeroth-order general Randi'c index, MATCH Commun. Math. Comput. Chem., 54 (2) (2005) 425-434.

14. J. Quadras, A. Sajiya Merlin Mahizl, I. Rajasingh, R. Sundara Rajan, Domination in certain chemical graphs, Journal of Mathematical Chemistry, 53 (2015) 207-219.

15. Vukicevic, D. and Furtula, B.: Topological index based on the ratios of geometrical and arithmetical means of end-vertex degrees of edges, J. Math. Chem., 46 (4) (2009) 1369-1376.

16. B. Bollobs, P. Erd"os, Graphs of extremal weights, Ars Combin., 50 (1998) 225-233.

17. D. Amic, D. Beslo, B. Lucic, S. Nikolic, N. Trinajstic, The vertex-connectivity index revisited, J. Chem. Inf. Comput. Sci., 38 (1998) 819-822.

18. A. Ilic, M. Ilic, Generalizations of Wiener polarity index and terminal Wiener index, Graphs Comb., 29 (2013) 1403-1416.

19. G. Caporossi, I. Gutman, P. Hansen, L. Pavlovic, Graphs with maximum connectivity index, Comput. Biol. Chem., 27 (2003) 85-90.

\section{Author' biography with Photo}

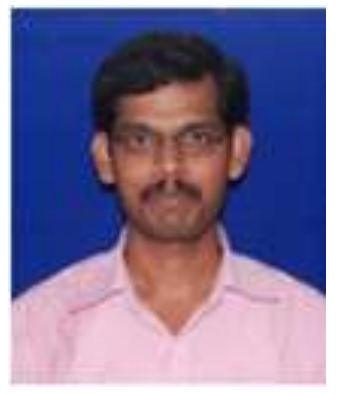

Dr. S. Prabhu is currently working as Assistant Professor of Department of Applied Mathematics, Sri Venkateswara College of Engineering (Autonomous), Sriperumbudur, India He Received his B.Sc., M.Sc., M.Phil. and Ph.D Form Loyola College (Autonomous), Chennai Affiliated to Madras University. During his B.Sc Course he received Rev. Fr. Alfrod Solomon Memorial Award for Securing highest Marks in the Major. During his Research Peroid, He received Rev. Fr. Racine Research Award. His Ph.D was Fully Sponsored by the Major Research Project No. F. 38$120 / 2009$ (SR) of the University Grants Commission, New Delhi, India. He also received the UNESCO-HP BGI Award given by Kuwait University Kuwait.

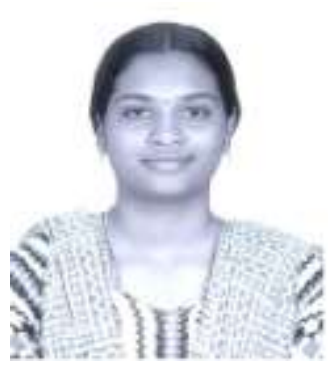

Mrs. M. Arulperumjothi is a Research Scholar in Department of Mathematics, Loyola College (Autonomous), Chennai. During her Post Graduation at Ethiraj College for Women (Autonomous), Chennai, she received UGC-IGSGC Fellowship No. F. 8-1/2014 -16/SGC-OBC2014-23907 / (SA-III), University Grants Commission, New Delhi, Government of India. 